\title{
AIP
}

\section{Drift velocity in non-isothermal inhomogeneous systems}

Mingcheng Yang and Marisol Ripoll

Citation: J. Chem. Phys. 136, 204508 (2012); doi: 10.1063/1.4723685

View online: http://dx.doi.org/10.1063/1.4723685

View Table of Contents: http://jcp.aip.org/resource/1/JCPSA6/v136/i20

Published by the American Institute of Physics.

\section{Additional information on J. Chem. Phys.}

Journal Homepage: http://jcp.aip.org/

Journal Information: http://jcp.aip.org/about/about_the_journal

Top downloads: http://jcp.aip.org/features/most_downloaded

Information for Authors: http://jcp.aip.org/authors

\section{ADVERTISEMENT}

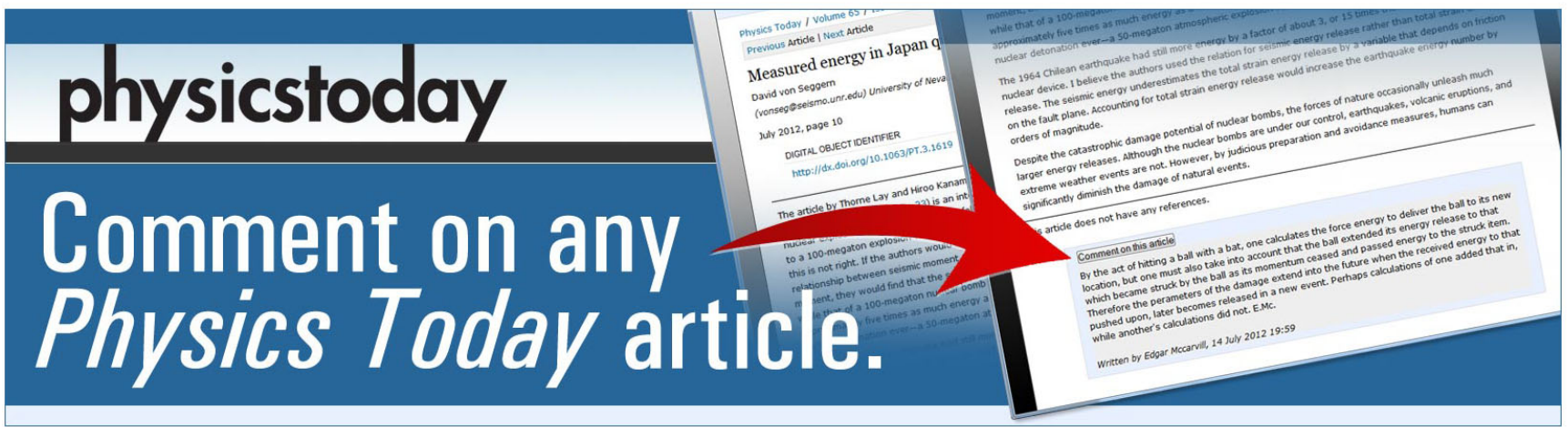




\title{
Drift velocity in non-isothermal inhomogeneous systems
}

\author{
Mingcheng Yanga) and Marisol Ripoll ${ }^{\text {b) }}$ \\ Theoretical Soft-Matter and Biophysics, Institute of Complex Systems, Forschungszentrum Jülich, \\ 52425 Jülich, Germany
}

(Received 12 December 2011; accepted 15 May 2012; published online 31 May 2012)

\begin{abstract}
Drift velocity and driving force are not directly proportional in the case of inhomogeneous suspensions, where a space dependent mobility induces an additional contribution to the drift velocity. Similarly, particle flux and drift velocity are related not only by the gradient of density but also by an additional contribution given by the gradient of the self-diffusion coefficient. We provide quantitative support to this scenario in a non-equilibrium system by means of computer simulations with a temperature gradient. Moreover, our simulation results demonstrate that the temperature gradientinduced mass transport coefficient, namely thermal diffusion coefficient, is not directly proportional to the drift velocity so that the well-accepted relation of proportionality is just an approximation.

(C) 2012 American Institute of Physics. [http://dx.doi.org/10.1063/1.4723685]
\end{abstract}

\section{INTRODUCTION}

The conceptual understanding of forces and drift velocities at a particle level is highly relevant when dealing with microscopic length scales. A force $\mathbf{f}$ acting on a particle suspended in a homogeneous solvent will induce a drift velocity $\mathbf{v}_{d}$, that precisely balances the viscous drag $\mathbf{v}_{d}=\mu \mathbf{f}$ at low Reynolds number, where $\mu$ is the mobility, and $\mu=1 / \zeta$ with $\zeta$ the friction coefficient. In fact, such relation is the basis of an advantageous experimental technique known as NFM (non-equilibrium force measurements), ${ }^{1-5}$ where the force is directly extracted from particle trajectories, which has been widely applied to colloid and polymer solutions. ${ }^{3,4}$ Nevertheless, real systems are not always homogeneous, and the related inhomogeneities fundamentally influence their dynamic behavior. This occurs, for example, in systems in confinement where the diffusion coefficient is known to be dependent on the particle distance to the wall. ${ }^{6}$ In such cases, it has been shown ${ }^{7}$ that there is an additional contribution to the drift velocity proportional to the gradient of diffusion coefficient,

$$
\mathbf{v}_{d}=\mu \mathbf{f}+\nabla D_{s}
$$

This means that the direct proportionality between the drift velocity and the driving force breaks down. NFM measurements of these systems, and the actual forces $\mathbf{f}$, can have mismatch in their magnitude and even their sign such that the additional term in Eq. (1) is necessary to obtain consistent results. Recently, the validity of Eq. (1) has been quantitatively verified by one of these NFM measurements, ${ }^{8,9}$ and previously also by an experiment with irregular confinement. ${ }^{10,11}$ In both cases though the use of Eq. (1) is justified by studying the related Langevin equation with the requirement of a particular interpretation of the multiplicative noise. However, the non-existence of an apparent Ito-Stratonovich dilemma which concerns the noise interpretation has already been clarified in the literature. ${ }^{7,12,13}$

\footnotetext{
a)Electronic mail: m.yang@fz-juelich.de.

b) Electronic mail: m.ripoll@ fz-juelich.de.
}

The previous considerations are valid for a large class of inhomogeneous systems with an equilibrium stationary state, for which the inhomogeneities arise from hydrodynamic interactions. ${ }^{7}$ This is for instance the case of particles close to a wall ${ }^{6,14}$ or two particles mutual diffusion. ${ }^{15}$ Nonetheless, there is another large class of inhomogeneous systems with a non-equilibrium steady state. Of particular relevance are systems where a position dependent self-diffusion coefficient originates from temperature inhomogeneities. Equation (1) has already been generalized, ${ }^{16}$ although its validity has not yet been verified in such systems. Furthermore, due to the development of single-particle tracking techniques, transport coefficients have been quantified by directly measuring the drift velocity, particularly the thermal diffusion coefficient of a dilute suspension. ${ }^{17}$ Therefore, it is critically important to establish a correct relation between the transport coefficients and the drift velocity for future applications.

In this paper, we explore the more general framework in which the drift velocity behavior is related to the inhomogeneous character of the system which can be isothermal or non-isothermal. A general drift-force relation is obtained using the van Kampen formula. ${ }^{18}$ This relation reduces to Eq. (1) for constant temperature. Simultaneously, an expression of the particle flux based on the drift velocity is obtained for the inhomogeneous system. We quantitatively check the validity of these relations in temperature gradient systems by performing non-equilibrium simulations. In terms of the validated expressions, we discuss the relation between the drift velocity and thermal diffusion coefficient.

\section{THEORETICAL DESCRIPTION}

Studies of inhomogeneous systems have received a considerable theoretical attention since the beginning of last century. ${ }^{7,16,18-21}$ However, there are still concerns about the existence of a universal description of such inhomogeneous systems, which implies that in principle each system needs to be studied individually. ${ }^{21,22}$ 
Our starting point is a minimal model derived by van Kampen from the extended Kramers equation ${ }^{18}$ valid in the linear response regime. The equation characterizes the particle flux $\mathbf{J}$ in a dilute particle dispersion with a temperature gradient

$$
\mathbf{J}(\mathbf{r})=n \mu(\mathbf{r}) \mathbf{f}-\mu(\mathbf{r}) \nabla\left[n(\mathbf{r}) k_{B} T(\mathbf{r})\right] .
$$

Here, $n$ is the particle concentration and $T$ the temperature. The force $\mathbf{f}$ was originally understood by van Kampen only as externally applied forces, but Widder and Titulaer ${ }^{20}$ extended this concept to consider as well mechanical driving forces exerted on single particles by the surrounding fluid. Equation (2) can be understood as a force balance equation on a volume element. ${ }^{23,24}$ The Brownian force $\nabla\left[n(\mathbf{r}) k_{B} T(\mathbf{r})\right]$ or gradient of the ideal gas osmotic pressure ${ }^{23,25}$ is not a real force and arises from the momentum change in the volume element due to particles entering into or departing from the volume element. This is in contrast to the mechanical driving force $n \mathbf{f}$ which is directly exerted on the particles of the volume element. The summation of these two forces balances then the friction force related to a particle flux by $\mathbf{J} / \mu$. In the stationary state with $\mathbf{J}=0$, Eq. (2) shows that the mechanical driving force is balanced by the Brownian force, which can also be seen in a multicomponent system from the equations of motion satisfied by each component. ${ }^{26}$ The steady-state Eq. (2) has also been validated by computer simulations. ${ }^{24}$ Alternatively, Eq. (2) can be understood as an extension of the Fick's law in the presence of a driving force and a temperature gradient. Originally, Eq. (2) was obtained for a dilute suspension of Brownian particles. However, when considering a tagged particle in a concentrated solution, the environment can be regarded as an effective solvent, such that Eq. (2) is correct by considering $\mathbf{f}$ and $\mu$ as the driving force and the mobility of the tagged particle, that include both solute-solvent and solute-solute contributions.

By adding and subtracting the term, $k_{B} T(\mathbf{r}) \nabla \mu(\mathbf{r})$, we can rewrite Eq. (2) in terms of the drift velocity as

$$
\mathbf{J}(\mathbf{r})=n(\mathbf{r}) \mathbf{v}_{d}-\nabla\left[n(\mathbf{r}) D_{s}(\mathbf{r})\right],
$$

with the drift velocity

$$
\mathbf{v}_{d}=\mu \mathbf{f}+k_{B} T \nabla \mu .
$$

Equation (3) has been written with the assumption that the local equilibrium approximation holds, and in particular the Einstein relation $D_{s}(\mathbf{r})=k_{B} T(\mathbf{r}) \mu(\mathbf{r})$ is valid. Further away from equilibrium, generalizations of the Einstein relation may eventually also be employed. ${ }^{27}$ Note that the self-diffusion coefficient $D_{s}(\mathbf{r})$ is related to the mean-squared-displacement or velocity autocorrelation function of a tagged particle, which is well defined in multicomponent systems. ${ }^{28,29}$ Differently, some authors exclusively use the name self-diffusion coefficient for single-component systems. ${ }^{30}$ In the presence of temperature gradients, the additional drift in Eq. (4) has been previously discussed for example by Landauer ${ }^{31}$ and by Bringuier. ${ }^{16}$ Equation (4) shows that the extra drift is proportional to the gradient of mobility rather than to the gradient of self-diffusion coefficient, which reduces to Eq. (1) in isothermal situations.
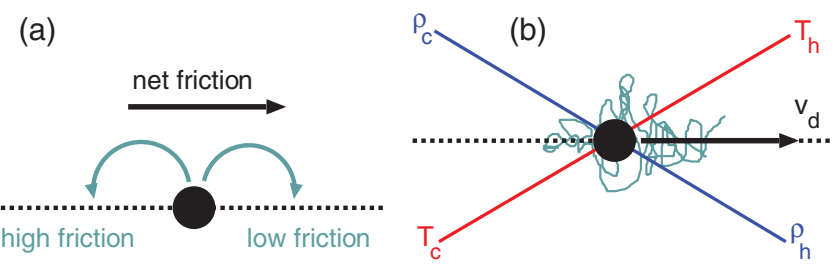

FIG. 1. (a) If a particle moves equally in all directions, an space-dependent friction coefficient leads to a net friction force towards lower friction areas. (b) Brownian particle drift in a temperature gradient.

Combining Eq. (3) with the continuity equation allows us to identify $\mathbf{v}_{d}$ with the drift velocity defined as

$$
\mathbf{v}_{d}=\frac{\partial}{\partial t} \int \mathbf{r} P(r, t) d V
$$

where $P(r, t)$ is the position distribution function of a tagged particle. This means that $\mathbf{v}_{d}$ can be quantified by evaluating the mean displacement of a single particle. It is important to emphasize that this drift velocity is related to diffusion and it should be understood on a Brownian time scale. Similar to the mean-squared-displacement, the drift velocity has a shorttime regime, and a long-time regime where it may reach a non-zero constant value.

Additionally, Eq. (3) shows the relation between the drift velocity $\mathbf{v}_{d}$ and the flux velocity $\mathbf{J}(\mathbf{r}) / n(\mathbf{r})$. The flux velocity concerns the net mass transport, that can be evaluated by averaging the instantaneous velocity of particles in a small volume element around $\mathbf{r}$. Equation (3) relates therefore the difference between the drift and the flux velocity to the spatial variation of the product of density and self-diffusion coefficient.

\section{A. Intuitive picture of the additional drift term}

From Eq. (4), it can be inferred that particle drift without mechanical driving force can occur when the friction is position dependent. In order to give an intuitive picture of this phenomenon, we first consider a suspension with a friction coefficient independent of position. If the particle moves faster in one particular direction, a net friction force appears in the opposite direction, such that a driving force is necessary to sustain the drift. We consider now a suspension with a position dependent friction. If the particle moves with the same preference in all directions (no drift), then a net friction force will appear towards the region with lower friction coefficient as shown in Fig. 1(a). An opposing driving force is then required to sustain the symmetric motion. In the absence of any mechanical driving force, the particle will therefore spontaneously drift towards areas with higher mobility (lower friction).

\section{SIMULATIONS WITH A TEMPERATURE GRADIENT}

In order to quantitatively test the validity of the previous theory in the non-isothermal systems, two types of simulations are performed. The first is a single Brownian particle with a position dependent temperature, and the second one is a binary mixture of Lennard-Jones particles. 


\section{A. Single Brownian particle}

First we study a single Brownian particle interacting with a thermal bath whose temperature $T(r)$ linearly varies in space (Fig. 1(b)). This means that the particle stochastically interchanges momentum and energy with an environment that has no structure apart from a position dependent temperature. To our knowledge, these are the first Brownian simulations combined with a temperature gradient.

Technically, the particle motion is implemented through a method known as random-multiparticle collision dynamics (MPC). This algorithm was proposed in Ref. 32 as a partner method of the MPC solvent, and it is therefore important to understand the relation between both methods. The MPC method properly takes into account the solvent induced hydrodynamic interactions by explicitly considering coarse grained solvent particles. ${ }^{33,34}$ However, the idea of randomMPC is to replace the explicit coarse grained solvent by a thermal bath, suppressing all solvent correlations. The Brownian particle is defined by its mass $M$, position $\mathbf{r}$, and velocity $\mathbf{v}$, and moves according to two alternating steps. In the streaming step, the particle moves ballistically during a collision time $h$, i.e., $\mathbf{r}(t+h)=\mathbf{r}(t)+h \mathbf{v}(t)$. In the collision step, the particle gets/liberates momentum and energy from/to a thermal bath, which is locally assumed to have a mass $M_{b}(r)$ and momentum $\mathbf{P}_{b}(r)$. This may be understood as the interaction with virtual particles from the thermal bath whose properties are position dependent. The collision consists of a stochastic rotation of the Brownian particle velocity relative to the center-of-mass velocity of the Brownian particle and the virtual particles, $\mathbf{v}_{c m}$, by an angle $\alpha$ around a random axis,

$$
\mathbf{v}(t+h)=\mathbf{v}_{c m}(t)+\mathcal{R}(\alpha)\left[\mathbf{v}(t)-\mathbf{v}_{c m}(t)\right],
$$

where $\mathcal{R}(\alpha)$ is the rotation matrix. The center-of-mass velocity is

$$
\mathbf{v}_{c m}=\frac{M \mathbf{v}+\mathbf{P}_{b}(r)}{M+M_{b}(r)}
$$

In standard MPC with explicit coarse grained solvent particles, $M_{b}(r)$ and $\mathbf{P}_{b}(r)$ refer to the total mass and momentum of the solvent particles within a collision cell. The local mass can further be expressed as $M_{b}(r)=m \rho(r)$ with $m$ the particle mass and $\rho(r)$ the number of particles in the collision cell, this can be understood as a dimensionless number density. The MPC solvent satisfies the ideal gas equation of state, such that $\rho(r)$ varies inversely with the temperature. ${ }^{33,35}$ However, in the random-MPC simulation, $\mathbf{P}_{b}(r)$ is directly selected from a Maxwell-Boltzmann distribution with variance $M_{b}(r) k_{B} T(r)$ with $k_{B}$ the Boltzmann constant and zero mean. The functional dependencies for $T(r)$, and $M_{b}(r)$ can be chosen. As illustrated in Fig. 1(b), in our simulations we opt for the same dependence as in standard MPC, namely $M_{b}(r) T(r)$ $=A$ with $A$ a constant factor. This choice implies that $\mathbf{P}_{b}(r)$ is in fact not position dependent, and that only $M_{b}(r)$ remains as position dependent. Simulations are performed with $M=5 \mathrm{~m}$, with $m$ the reference mass, $\alpha=130^{\circ}$, the mean temperature $k_{B} \bar{T}=1, A=5.25$, and varying $h$. These values are similar to those in related simulations with the standard MPC model. ${ }^{35}$ The employed parameters are related with the coupling of the Brownian particle and the thermal bath, and determine the thermal bath properties such as the friction coefficient. ${ }^{32}$ Therefore, in contrast to what the particular choice of $\mathrm{M} / \mathrm{m}$ may seem to indicate, these parameters do not reflect other microscopic information, such that the masses of the Brownian particle and the microscopic underlying solvent particles could still be separated by orders of magnitude. In the simulation results, time and length are scaled in terms of $\sqrt{k_{B} \bar{T} / m a^{2}}$ and $a$, respectively, with $a$ the reference length.

Apart from the technical details of its particular implementation, random-MPC has to be understood as the interaction with a thermal bath, similar to a standard Brownian dynamics simulation (BD). The main difference is that in BD frictional and stochastic forces are imposed, with coefficients that satisfy by construction the Einstein relation. In randomMPC only the stochastic collision with virtual particles is imposed. Friction and thermal fluctuations are a consequence of such collision rule, and the Einstein relation can be proved to be automatically satisfied (see Appendix). Particularly interesting in random-MPC is that the transport properties can be directly compared with standard MPC, and especially that inhomogeneities are easily taken into account.

The mean displacement of a Brownian particle $\Delta \mathbf{r}(t)$ $=\mathbf{r}(t)-\mathbf{r}(0)$ can then be straightforwardly obtained. An example is displayed in Fig. 2(a) for different directions. Single trajectories are changing direction wildly such that averaging over many realizations is necessary. In the direction of the gradient, the averaged displacement is linearly increasing towards the warm direction, while it is vanishing in the two perpendicular directions. The slope of $\Delta \mathbf{r}$ with $t$ determines $\mathbf{v}_{d}$. Figure 2(b) presents values of the measured drift velocities, showing a linear dependence with the temperature gradient and the random-MPC collision time.
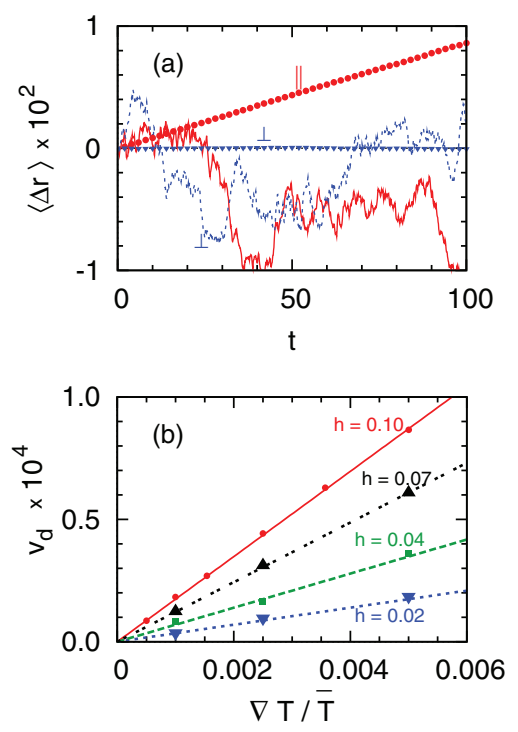

FIG. 2. (a) Particle displacement as a function of time, parallel and perpendicular to the temperature gradient, with $h=0.1$ and $\nabla T / \bar{T}=0.005$. Positive displacement goes to hot. Lines are single trajectories downscaled by a factor 500, symbols are averaged over $10^{9}$ trajectories. (b) Drift as a function of $\nabla T$ for various $h$ values. Symbols are simulation results and lines theoretical predictions in Eq. (9). 
In order to test Eq. (4), the quantities on the right side need to be quantified, i.e., $\mathbf{f}$ and $\mu$. In principle, they could be directly computed in simulations. However, for the randomMPC model this is not necessary, since both quantities can be analytically exactly obtained. First, the mechanical driving forces, $\mathbf{f}$, exerted on the Brownian particle are zero by construction. This can be seen since the bath precisely follows the local Maxwell-Boltzmann distribution (exact local equilibrium), which implies that the non-equilibrium mechanical driving force ${ }^{36}$ induced by the deviation of the distribution function from the local Maxwell-Boltzmann distribution, is completely vanishing. Consequently, the first term on the right side of Eq. (4) vanishes such that the non-vanishing values shown in Fig. 2 already prove the existence of an extra drift contribution non-proportional to the driving force. Second, the simple collision rule in the random-MPC allows us to analytically calculate the expression of the mobility of the Brownian particle in the random-MPC (see calculation details in the Appendix),

$$
\mu(r)=\frac{h}{M}\left[\frac{1}{\gamma_{\alpha} \gamma_{b}(r)}-\frac{1}{2}\right],
$$

where $\gamma_{\alpha}=2(1-\cos \alpha) / 3$ and $\gamma_{b}(r)=1-M /\left[M_{b}(r)+M\right]$.

Thus, the analytical prediction of $\mathbf{v}_{d}$ can be obtained in terms of Eq. (8) as

$$
\mathbf{v}_{d}=\frac{h}{A \gamma_{\alpha}} k_{B} T \nabla T .
$$

The linear dependence of $\mathbf{v}_{d}$ with $\nabla T$ and $h$ observed in Fig. 2(b) is then explained by Eq. (9). Equation (4) can be tested by comparing the drift velocity obtained in the simulations with the analytical prediction, which has no adjustable parameters. In Fig. 2(b), the measured drift velocities for several temperature gradients and simulation parameters are displayed together with their theoretical predictions showing a perfect agreement. The agreement is so good since the approximations in the analytical calculation are exactly fulfilled for this example, and the single-particle simulations can be performed with very high statistical accuracy.

On the other hand, the fact that mechanical driving force exerted on the Brownian particle is zero, implies that the Brownian particle obeys the ideal gas equation of state, such that the density profile that is imposed for the thermal bath and sketched in Fig. 1(b) happens to apply as well to the Brownian particle. It could be therefore counter-intuitive to note that drift velocity occurs in the direction where the Brownian particle has a lower concentration.

Random-MPC offers us an ideal opportunity to study the drift velocity induced by the non-uniform mobility term, since the mechanical driving force is completely vanishing. The drift velocity relation in Eq. (4) though is much more general since it is valid as long as the van Kampen equation in Eq. (2) applies. If instead of random-MPC, another system would be chosen, the exact local thermal equilibrium could not be completely fulfilled and then the deviation from the Maxwell-Boltzmann distribution would produce a non-equilibrium mechanical driving force on the suspended particle. $^{36,37}$ Thus, besides the non-uniform mobility, the mechanical driving force would also contribute to the drift velocity. Therefore Eq. (4) would still be valid, although the evaluation of the related quantities would become more involved.

\section{B. Systems of Lennard-Jones particles}

In the second simulation example, we consider a nonequilibrium case with nonzero mechanical driving force. In this case, we precisely verify Eq. (3) in the stationary state, which together with the validation of the steady-state Eq. (2) presented in Ref. 24 completes the quantitative verification of Eq. (4). We study systems of Lennard-Jones particles composed by one or two components with linear temperature profile. In the case of a molecular mixture, Eqs. (2)-(4) apply to each component, when the observables are related to the corresponding component. In a Lennard-Jones system, particles $i$ and $j$ at a distance $r_{i j} \leq 2.5 \sigma_{i j}$ interact via

$$
U_{L J}\left(r_{i j}\right)=4 \epsilon_{i j}\left[\left(\frac{\sigma_{i j}}{r_{i j}}\right)^{12}-\left(\frac{\sigma_{i j}}{r_{i j}}\right)^{6}\right],
$$

where $\epsilon_{i j}$ is the potential depth and $\sigma_{i j}$ the particle diameter. This system has extensively been studied in the presence of temperature gradients, ${ }^{38-40}$ although the focus has always been to determine the Soret coefficient instead of the drift velocity as in the present work. The particle masses are set equal $m_{11}=m_{22}$, and the interaction parameters between unlike particles are determined by the Lorentz-Berthelot mixing rules, $\epsilon_{12}=\sqrt{\epsilon_{11} \epsilon_{22}}$, and $\sigma_{12}=\left(\sigma_{11}+\sigma_{22}\right) / 2$. Quantities are expressed in reduced units, i.e., $m_{22}, \sigma_{22}$, and $\epsilon_{22}$ are set to 1 , with $T^{*}=k_{B} T / \epsilon_{12}, \rho^{*}=\rho \sigma_{12}^{3}$. The typical system size is $60 \sigma_{22}$ in the $\nabla T$ direction and $12.5 \sigma_{22}$ in the perpendicular one.

The temperature gradient is imposed by employing boundary-driven non-equilibrium molecular dynamics. This consist of defining a cold layer at one boundary, and a parallel hot layer at the center, together with periodic boundary conditions. An energy flux is then externally imposed from the cold to the hot layer, which translates into an opposite energy flux and a temperature gradient in between such layers. ${ }^{41,42}$ The mean temperature remains fixed by using the Berendsen thermostat. In the steady state, there is no mass flux, and $\mathbf{v}_{d}, n(r)$ and $T(r)$ can be directly measured. It should be emphasized that in the steady state although the flux velocity is vanishing, the drift velocity still can be independently determined. The displacement is calculated by tracking the trajectory of particles randomly chosen from the region far away from the boundaries. The tracking time must be small enough for the particle not to be able to arrive to the cold or warm boundaries, but large enough to reach the diffusive time regime. The averaged particle displacement of species 1 in the $\mathrm{LJ}$ gas mixture in Fig. 3(a) shows to increase linearly in the direction parallel to the temperature gradient and not in the perpendicular ones, such that $\mathbf{v}_{d}$ can be determined. In Fig. 3(a) it can be observed that for short times, the diffusive time scale has not been reached, and the drift velocity still varies with time.

In order to quantitatively verify Eq. (3) the gradient of the self-diffusion coefficient has to be determined. $D_{s}$ is obtained by measuring the particle mean-squared-displacement 

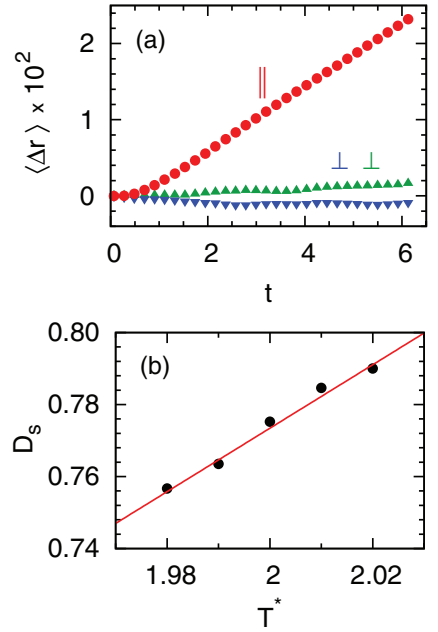

FIG. 3. Results for particles of species 1 in equimolar LJ gas mixture (quantities expressed in reduced units and parameters in Table I). (a) Particle mean displacement averaged over $3 \times 10^{7}$ trajectories as a function of time. (b) Self-diffusion coefficient versus temperature. Symbols are simulation results and the line is a linear fit.

in separate equilibrium simulations in the isothermal-isobaric ensemble at different imposed temperatures, and varying particle molar fractions that corresponds to the local quantities of the non-equilibrium simulation. Performing these equilibrium simulations is meaningful since the temperature gradients employed in the non-isothermal simulations are small enough such that the local equilibrium approximation can be applied. Results displayed in Fig. 3(b) show a linear increase of the self-diffusion with temperature from which $\nabla D_{s}$ can be calculated. With this, the three contributions in Eq. (3) have been independently measured such that the expression can be quantitatively verified. Simulation parameters and results are summarized in Table I and show that Eq. (3) is very convincingly satisfied within the error of the measurements. The results of the equimolar mixtures imply that Eq. (3) is valid for both diluted and concentrated solutions. This is relevant since van Kampen derived Eq. (2) for a dilute Brownian particle suspension where $\mu$ referred to the mobility of a single particle in a background random solvent. However, when considering a tagged particle in a concentrated solution, like an equimolar liquid mixture of Lennard-Jones particles, the environment can be regarded as an effective solvent. Equations (2)-(4) are then correct by understanding all quantities referred to those

TABLE I. Drift velocity measurements in LJ systems obtained from direct measurement and from the calculation of the gradients. Results correspond to: (a) single component gas with mean temperature $T^{*}=2$ and density $\rho^{*}$ $=0.26$. (b) gas equimolar mixture with $T^{*}=2$ and $\rho^{*}=0.34$. The parameter ratios are $\sigma_{11}=1.2 \sigma_{22}$ and $\epsilon_{11}=\epsilon_{22} . \mathrm{s} 1$, s 2 stand respectively for species 1 and 2. c) liquid equimolar mixture with $T^{*}=0.85, \rho^{*}=0.81, \sigma_{11}=\sigma_{22}$, and $\epsilon_{11}=2 \epsilon_{22}$.

\begin{tabular}{|c|c|c|}
\hline System & $\mathbf{v}_{d} \times 10^{3}$ & $\nabla\left(D_{s} n\right) / n \times 10^{3}$ \\
\hline (a) pure gas & $6.5(3)$ & $6.1(7)$ \\
\hline (b) gas mixt. s1 & $4.2(3)$ & $3.9(6)$ \\
\hline gas mixt. s2 & $5.1(5)$ & $4.8(4)$ \\
\hline (c) liquid mixt. s1 & $0.92(9)$ & $1.00(8)$ \\
\hline liquid mixt. s2 & $2.2(2)$ & $2.2(2)$ \\
\hline
\end{tabular}

of a tagged particle, and in particular $D_{s}$ related to the mean square displacement of the tagged particle.

From the results in Table I, various conclusions can be drawn. First, the single-component gas result in Table I (a), confirms Brenner's prediction about the existence of a drift velocity in a steady-state single-component solvent. ${ }^{43}$ Independently from the volume transport theory presented in Ref. 43, the result can be straightforwardly understood from Eq. (3) since all related quantities are uniquely defined independent of the number of components. Second, all the presented drift velocities occur in the direction of increasing temperatures. In these systems the gradient of mobility points always to the warm side. These results seem to indicate that this contribution is more important than the related mechanical forces in Eq. (4). A different situation would be in case of systems such as colloidal suspensions where the mechanical driving forces can be dominant and point to both directions depending on the particular interactions. And finally, the fact that the agreement shown in Table I applies to both components in the mixtures, constitutes an example of the validity of Eq. (3) in systems both with positive and negative thermophoresis.

\section{THERMOPHORETIC VELOCITY}

One of the most relevant phenomena appearing in the presence of a temperature gradient is known as thermal diffusion or Soret effect and it refers to the mass flux induced in fluid mixtures by the temperature gradient. ${ }^{44,45}$ The effect is ubiquitous in non-isothermal fluid mixtures, such as colloidal suspensions and molecular mixtures. In this section, we establish a relation between the drift velocity and the thermal diffusion coefficient of a dilute solution through the particle flux Eq. (3), which is essential for experimentally determining the thermal diffusion coefficient from the single-particle tracking. ${ }^{17}$ For binary mixtures, the particle flux can be phenomenologically expressed as,

$$
\mathbf{J}=-\tilde{n} D_{m} \nabla x-\tilde{n} x(1-x) D_{T} \nabla T .
$$

This is also the standard definition of the thermal diffusion coefficient $D_{T} . D_{m}$ is the mutual diffusion coefficient, and $S_{T}=D_{T} / D_{m}$ is the so-called Soret coefficient. $x=n / \tilde{n}$ is the solute mole fraction, and here, $\tilde{n}=n+n^{\prime}$ is the total number density, being $n$ and $n^{\prime}$ the number density of the two components. In the dilute case $\left(n \ll n^{\prime}\right.$ and $\left.D_{m} \simeq D_{s}\right),{ }^{28}$ the comparison of Eqs. (3) and (11) leads to,

$$
D_{T} \nabla T=\nabla D_{s}-D_{s} \beta \nabla T-\mathbf{v}_{d},
$$

with $\beta=\left(-1 / n^{\prime}\right) \partial n^{\prime} / \partial T$ the solvent thermal expansion coefficient. This expression has already been obtained by Bringuier in Ref. 46 following an alternative route.

The thermophoretic velocity $\mathbf{v}_{T}$ defined just as,

$$
\mathbf{v}_{T} \equiv-D_{T} \nabla T
$$

is of standard use in the literature. ${ }^{17,45,47}$ Nevertheless, Eq. (12) shows that $\mathbf{v}_{T}$ does not exactly correspond to the measurable drift velocity $\mathbf{v}_{d}$. The expression $\mathbf{v}_{T} \simeq \mathbf{v}_{d}$ can just be considered as a good approximation only when $\left|D_{T}\right| \gg$ $\left|d D_{s} / d T-D_{s} \beta\right|$, which is indeed the case in most complex 
fluids. ${ }^{17,47,48}$ In a recent work, ${ }^{49}$ we propose a model to simulate a colloid in a temperature gradient. The importance of these two additional terms is quantified to be $0.5 \%$ of the last term within the employed model and parameters. Nevertheless, for colloidal suspensions with low $D_{T} / D_{S}$ (particularly, in the region of $D_{T}$ changing sign), low-weight polymer solutions or molecular mixtures, the first two terms on the right side of Eq. (12) are comparable with $\mathbf{v}_{d}$ and even larger, and thus need to be considered. Because Eq. (3) used to obtain Eq. (12) has been quantitatively verified in Sec. III, our simulation results present a quantitative support to Eq. (12).

Finally, we want to emphasize that Eq. (12) is valid only in the dilute regime. However, the drift velocity will not be directly proportional in general to the thermal diffusion coefficient in the concentrated regime. Our results for the LJ mixture in Table I constitute a very clear example. By definition, the Soret coefficient of the two components of a mixture are equal with opposite signs, $S_{T, 1}=-S_{T, 2}$, such that the two corresponding $D_{T}$ will also have opposite signs. The velocity $\mathbf{v}_{d}$ has though the same direction in both cases, which implies that $\mathbf{v}_{d}$ and $\mathbf{v}_{T}$ have even opposite directions for one of these components.

\section{DISCUSSION AND CONCLUSIONS}

The validity of the relation between drift velocity and mechanical driving force stated in Eq. (4) has been investigated in Sec. III by means of computer simulations in nonisothermal suspensions. For inhomogeneous systems with uniform temperature, Eq. (4) straightforwardly reduces to Eq. (1). As mentioned in the Introduction, Eq. (1) has been quantified by a recent experiment of colloidal dispersion, ${ }^{8,9}$ in which the temperature of the system is uniform and the inhomogeneity is due to hydrodynamic interactions between the particles and the boundary wall. ${ }^{6,14}$ In that experiment, ${ }^{8,9}$ Volpe et al. measure both the mechanical forces exerted on the particle and the resultant total drift velocity, from which the extra drift velocity term is characterized. The extra drift velocity perfectly agrees with $\nabla D_{s}$ obtained from theoretical calculation. ${ }^{6}$ Therefore, Eq. (4) embraces the description of all isothermal and non-isothermal inhomogeneous cases, such that it can be regarded as the most generalized drift-force relation in inhomogeneous suspensions, as long as the system is in the linear response regime.

Drift without or against particle accumulation can occur in inhomogeneous suspensions. These interesting and even counter-intuitive behaviors can be understood in terms of the particle flux equation involving the drift velocity, i.e., Eq. (3). In a homogeneous system without flux, a non-vanishing drift velocity is necessarily accompanied by a particle accumulation in the same direction. This occurs for example in a sedimentation system confined in the gravity direction. ${ }^{50}$ However, in an inhomogeneous system, the particle flux arising from the drift velocity may precisely cancel with the one due to the gradient of self-diffusion coefficient in Eq. (3), which translates into a drift in the absence of particle accumulation. This is indeed the case in the irregular confinement system of Lançon et al. ${ }^{10}$ In their experiments, a colloidal suspension is confined between wedged walls without suffering any me- chanical driving force. This geometry produces an increase of the self-diffusion coefficient with separation between walls. In the stationary state, a drift velocity of the individual particles is observed in the direction of the diffusion coefficient gradient, but with constant concentration. In the simulations with temperature gradients presented in this work, the concentration of the particles is mostly larger in the region of low temperature, e.g., in the case of the Brownian particle and the LJ gas, while the related drift velocities point to the hot area. Therefore, this constitutes an example where the drift occurs in the opposite direction to the particle accumulation.

All the simulations presented in this work are performed in steady states. Nevertheless, we expect that Eqs. (3) and (4) are still valid in unsteady states, as long as the typical relaxation time of the system is larger than the diffusive time scale. Furthermore, our results broaden the validity of theory to systems in which all components have similar sizes and masses, such as molecular mixtures. This is since the original framework in Eq. (2) was postulated for purely Brownian motion, where solute and solvent have related scales separated by orders of magnitude. Conversely, simulations with colloidal suspensions are also expected to confirm Eqs. (3) and (4).

In summary, this paper revisits the fundamental concept of extra drift that appears in systems where the self-diffusion or mobility are spatially varying. In the presence of temperature gradients, two types of simulations allow us to quantitatively support the theoretical framework for the first time in non-equilibrium and to broaden its validity. First we propose a method to combine a Brownian simulation with a temperature gradient. The Brownian particle shows the appearance of a drift velocity towards decreasing friction and warmer areas in the absence of any mechanical driving force. Second, standard simulations of Lennard-Jones particles in the absence of mass flux verify the relation between the drift velocity and the gradients of density and self-diffusion coefficient in Eq. (3), in a situation where the Einstein relation is valid. In a recent work, ${ }^{24}$ we further verify the steady-state force balance condition in Eq. (2), which together with the actual results complete the quantitative verification of Eq. (4). As a consequence of the validated expression for the mass flux in Eq. (3), it can be demonstrated that the thermophoretic velocity is not necessarily equivalent to the measurable drift velocity, albeit this is widely accepted. Due to the development of microscale single-particle tracking techniques, the correct understanding of the drift velocity in isothermal and non-isothermal inhomogeneous systems appears then to be of high importance from a fundamental and technological viewpoints.

\section{ACKNOWLEDGMENTS}

The authors warmly acknowledge useful discussions with E. Bringuier, A. Würger, D. Lüsebrink, J. K. G. Dhont, S. Wiegand, P. R. Lang, G. Nägele, J. M. Rubí, and G. A. Vliegenthart.

\section{APPENDIX: EINSTEIN RELATION FOR RANDOM MPC}

In this appendix we calculate the particle mobility $\mu$ and prove the Einstein relation for a particle interacting with a 
random-MPC solvent. The motion of a Brownian particle of mass $M$ and velocity $\mathrm{V}$ can be described by the Langevin equation as,

$$
M \dot{\mathrm{v}}=-\frac{1}{\mu} \mathrm{v}+\mathbf{f}_{\xi},
$$

with $\mathbf{f}_{\xi}$ the stochastic force. In case the time evolution is discretized with a collision time $h$, as in random-MPC, the continuous acceleration $\dot{v}$ can be approximated in terms of the discrete pre- and post-collision velocities as

$$
\dot{\mathbf{v}}(t+h / 2)=\frac{1}{h}[\mathbf{v}(t+h)-\mathbf{v}(t)],
$$

and the corresponding velocity reads

$$
\mathbf{v}(t+h / 2)=\frac{1}{2}[\mathbf{v}(t+h)+\mathbf{v}(t)] .
$$

Inserting Eqs. (A2) and (A3) into (A1), we have

$$
\frac{M}{h}[\mathbf{v}(t+h)-\mathbf{v}(t)]=-\frac{1}{2 \mu}[\mathbf{v}(t+h)+\mathbf{v}(t)]+\mathbf{f}_{\xi},
$$

In order to obtain $\mu$, the collisional average is taken in both sides of Eq. (A4),

$$
\frac{M}{h}\langle\mathbf{v}(t+h)-\mathbf{v}(t)\rangle=-\frac{1}{2 \mu}\langle\mathbf{v}(t+h)+\mathbf{v}(t)\rangle,
$$

since $\left\langle\mathbf{f}_{\xi}\right\rangle$ vanishes. The collisional average of the randomMPC includes averaging over the orientation of the random rotation and over the momentum distribution of the virtual particles. To calculate the averages in Eq. (A5), the MPC collision rule in Eq. (6) is employed

$$
\langle\mathbf{v}(t+h)-\mathbf{v}(t)\rangle=\left\langle[\mathcal{R}(\alpha)-I]\left[\mathbf{v}(t)-\mathbf{v}_{c m}(t)\right]\right\rangle,
$$

with $I$ the unity matrix. The rotational average of an arbitrary vector $\mathbf{A}$ can be calculated by geometrical arguments for rotations in three dimensions around an arbitrary axis to be

$$
\langle(\mathcal{R}(\alpha)-I) \mathbf{A}\rangle=-\gamma_{\alpha}\langle\mathbf{A}\rangle,
$$

with $\gamma_{\alpha}=2(1-\cos \alpha) / 3$. Considering the definition of $\mathbf{v}_{c m}$ for the random-MPC in Eq. (7), and calculating the momentum average we obtain,

$$
\langle\mathbf{v}(t+h)-\mathbf{v}(t)\rangle=-\gamma_{\alpha} \gamma_{b}\langle\mathbf{v}(t)\rangle,
$$

with $\gamma_{b}=M_{b} /\left(M+M_{b}\right)$. Similarly,

$$
\langle\mathbf{v}(t+h)+\mathbf{v}(t)\rangle=\left(2-\gamma_{\alpha} \gamma_{b}\right)\langle\mathbf{v}(t)\rangle .
$$

Substituting now Eqs. (A8) and (A9) into (A5), we finally obtain the mobility

$$
\mu=\frac{h}{M} \frac{2-\gamma_{\alpha} \gamma_{b}}{2 \gamma_{\alpha} \gamma_{b}} .
$$

It can be seen that the expression in Eq. (A10) is just Eq. (8), where the position dependence of $M_{b}(r)$ and therefore of $\mu(r)$ have also been accounted for.

The self-diffusion coefficient of a heavy particle in a MPC-fluid has been calculated ${ }^{34,51}$ from the Green-Kubo formalism $D=1 / 3 \int_{0}^{\infty} d t\langle\mathbf{v}(t) \mathbf{v}(0)\rangle$ by assuming molecular chaos and following a similar averaging procedure as here. The result can be expressed as $D=k_{B} T \mu$, with $\mu$ in
Eq. (8). The molecular chaos assumption neglects all correlations of the interacting solvent at different times. This assumption has shown to be imprecise ${ }^{34,52}$ for the standard MPC solvent where hydrodynamic interactions are of importance, but it is by construction exact in random-MPC. This means that the Einstein relation is proved to be exactly fulfilled in random-MPC. For standard MPC, this would also be the case if the molecular chaos assumption holds, but it would not provide any additional information otherwise.

${ }^{1}$ P. Poulin, V. Cabuil, and D. A. Weitz, Phys. Rev. Lett. 79, 4862 (1997).

${ }^{2}$ J. C. Neto, R. Dickman, and O. N. Mesquita, Physica A 345, 173 (2005).

${ }^{3}$ S. K. Sainis, V. Germain, and E. R. Dufresne, Phys. Rev. Lett. 99, 018303 (2007)

${ }^{4}$ P. Wu, R. Huang, C. Tischer, A. Jonas, and E.-L. Florin, Phys. Rev. Lett. 103, 108101 (2009).

${ }^{5}$ J. W. Merrill, S. K. Sainis, and E. R. Dufresne, Phys. Rev. Lett. 103, 138301 (2009)

${ }^{6}$ H. Brenner, Chem. Eng. Sci. 16, 242 (1961).

${ }^{7}$ A. W. Lau and T. C. Lubensky, Phys. Rev. E 76, 011123 (2007).

${ }^{8}$ G. Volpe, L. Helden, T. Brettschneider, J. Wehr, and C. Bechinger, Phys. Rev. Lett. 104, 170602 (2010).

${ }^{9}$ T. Brettschneider, G. Volpe, L. Helden, J. Wehr, and C. Bechinger, Phys. Rev. E 83, 041113 (2011).

${ }^{10}$ P. Lançon, G. Batrouni, L. Lobry, and N. Ostrowsky, Europhys. Lett. 54, 28 (2001).

${ }^{11}$ P. Lançon, G. Batrouni, L. Lobry, and N. Ostrowsky, Physica A 304, 65 (2002).

${ }^{12}$ N. van Kampen, Stochastic Processes in Physics and Chemistry (NorthHolland, Amsterdam, 1992), Chap. IX and X.

${ }^{13}$ J. M. Sancho, Phys. Rev. E 84, 062102 (2011)

${ }^{14}$ P. Holmqvist, J. K. G. Dhont, and P. R. Lang, Phys. Rev. E 74, 021402 (2006).

${ }^{15}$ J. C. Crocker, J. Chem. Phys. 106, 2837 (1997).

${ }^{16}$ E. Bringuier and A. Bourdon, J. Non-Equil. Thermodyn. 32, 221 (2007).

${ }^{17}$ S. Duhr and D. Braun, Proc. Natl. Acad. Sci. U.S.A. 103, 19678 (2006).

${ }^{18}$ N. van Kampen, J. Phys. Chem. Solids 49, 673 (1988).

${ }^{19}$ S. Chapman, Proc. R. Soc. London, Ser. A 119, 34 (1928).

${ }^{20}$ M. E. Widder and U. M. Titulaer, Physica A 154, 452 (1989).

${ }^{21}$ M. J. Schnitzer, Phys. Rev. E 48, 2558 (1993)

${ }^{22}$ N. van Kampen, Z. Phys. B 68, 135 (1987).

${ }^{23}$ J. K. G. Dhont, J. Chem. Phys. 120, 1642 (2004).

${ }^{24}$ M. Yang and M. Ripoll, J. Phys. Condens Matter 24, 195101 (2012).

${ }^{25}$ F. Jülicher and J. Prost, Eur. Phys. J. E 29, 27 (2009).

${ }^{26}$ R. Bearman and J. Kirkwood, J. Chem. Phys. 28, 136 (1958).

${ }^{27}$ D. Rings, R. Schachoff, M. Selmke, F. Cichos, and K. Kroy, Phys. Rev. Lett. 105, 090604 (2010).

${ }^{28}$ G. Jacucci and I. McDonald, Physica A 80, 607 (1975).

${ }^{29}$ D. L. Jolly and R. J. Bearman, Mol. Phys. 41, 137 (1980).

${ }^{30}$ R. B. Bird, W. E. Stewart, and E. N. Lightfoot, Transport Phenomena, 2nd ed. (Wiley, 2002).

${ }^{31}$ R. Landauer, J. Stat. Phys. 53, 233 (1988).

${ }^{32}$ M. Ripoll, R. G. Winkler, and G. Gompper, Eur. Phys. J. E 23, 249 (2007).

${ }^{33}$ A. Malevanets and R. Kapral, J. Chem. Phys. 110, 8605 (1999).

${ }^{34}$ M. Ripoll, K. Mussawisade, R. G. Winkler, and G. Gompper, Phys. Rev. E 72, 016701 (2005).

${ }^{35}$ D. Lüsebrink and M. Ripoll, J. Chem. Phys. 136, 084106 (2012).

${ }^{36}$ E. Bringuier and A. Bourdon, Physica A 385, 9 (2007).

${ }^{37} \mathrm{~S}$. Chapman and T. Cowling, The Mathematical Theory of Non-uniform Gases (Cambridge University Press, Cambridge, 1939).

${ }^{38}$ D. Reith and F. Müller-Plathe, J. Chem. Phys. 112, 2436 (2000).

${ }^{39}$ G. Galliéro, B. Duguay, J.-P. Caltagirone, and F. Montel, Fluid Phase Equilib. 208, 171 (2003).

${ }^{40}$ P. A. Artola and B. Rousseau, Phys. Rev. Lett. 98, 125901 (2007).

${ }^{41}$ B. Hafskjold, T. Ikeshoji, and S. K. Ratkje, Mol. Phys. 80, 1389 (1993).

${ }^{42}$ F. Müller-Plathe, J. Chem. Phys. 106, 6082 (1997).

${ }^{43}$ H. Brenner, Phys. Rev. E 74, 036306 (2006). 
${ }^{44}$ S. Wiegand, J. Phys.: Condens. Matter 16, R357 (2004).

${ }^{45}$ R. Piazza and A. Parola, J. Phys.: Condens. Matter 20, 153102 (2008).

${ }^{46}$ E. Bringuier, Phil. Mag. 87, 873 (2007).

${ }^{47}$ A. Würger, Rep. Prog. Phys. 73, 126601 (2010).

${ }^{48}$ D. Stadelmaier and W. Köhler, Macromolecules 41, 6205 (2008).
${ }^{49}$ D. Lüsebrink, M. Yang, and M. Ripoll, "Thermophoresis of colloids by mesoscale simulations," J. Phys.: Condens. Matter (in press).

${ }^{50}$ W. Russel, D. Saville, and W. Schowalter, Colloidal dispersions (Cambridge University Press, 1995).

${ }^{51}$ E. Tüzel, M. Strauss, T. Ihle, and D. M. Kroll, Phys. Rev. E 68, 036701 (2003).

${ }^{52}$ E. Tüzel, T. Ihle, and D. M. Kroll, Phys. Rev. E 74, 056702 (2006). 